\title{
La traduction italienne de Les Soleils des indépendances d'Ahmadou Kourouma : le cas du traitement des diatopismes
}

\author{
Chiara Brandolini \\ Università degli Studi di Pavia- Université Paris IV Sorbonne
}

\section{Introduction}

L'article présente l'analyse effectuée sur la traduction italienne de Les Soleils des indépendances d'Ahmadou Kourouma. Une brève analyse des éléments péritextuels qui influencent la réception du roman dans la culture cible précède une véritable analyse linguistique. Les diatopismes présents dans l'œuvre, qui représentent la principale trace visible de la situation plurilingue de la Côte-d'Ivoire, ont été classifiés suivant des méthodes lexicographiques : après avoir vérifié leur caractère régional dans les dictionnaires du français régional de la Côte-d'Ivoire et de l'Afrique noire (en particulier les dictionnaires de Lafage, Duponchel, et l'IFA), les diatopismes ont été classés suivant un tableau conçu en référence aux diatopismes du Canada par Poirier (Poirier 43), remanié par Thibault (Thibault section Cours de francophonie $3^{e}$ année de licence, introduction.). Ensuite, l'analyse des techniques de traduction employées dans l'œuvre cible a été mise en place.

\section{1) Éléments péritextuels}

Pour mieux comprendre comment l'œuvre se situe dans la culture française et francophone et dans la culture italienne, la prise en compte des éléments péritextuels (maison d'édition, présence ou absence de préfaces et postfaces, supports et présentation) est nécessaire. Les maisons d'éditions et leurs politiques éditoriales sont centrales pour comprendre la façon dont l'œuvre s'insère dans le panorama littéraire d'un pays. La place de la littérature étrangère dans la maison d'édition et son insertion dans la littérature générale ou dans une collection particulière, sont symptomatiques de la conception de l'œuvre traduite dans le système culturel d'arrivée. En effet, le choix implique une conception de la littérature qui influence de manière implicite et explicite le lecteur. Toute œuvre en traduction se situe, suivant les politiques éditoriales des maisons d'éditions (Risterucci-Roudnicky 21), au croisement entre deux axes qui déterminent la place symbolique de l'œuvre importée : d'un côté, l'axe horizontal représente la distance de l'œuvre étrangère par rapport à la culture-cible et tend à la naturalisation, de l'autre côté, l'axe vertical de la canonisation représente la nouveauté et la découverte de l'œuvre. Ainsi, une collection spécifique pour la littérature étrangère souligne les particularités hybrides de l'œuvre traduite. La prise en compte du profil de l'éditeur, de la collection et du péritexte éditorial qui en soulignent l'origine et les spécificités culturelles devient essentielle.

Le roman analysé est paru en France aux Editions du Seuil qui, née dans les années 30, est une ancienne maison généraliste qui a publié dans tous les domaines. Même si la maison se déclare fidèle aux origines et reste liée au brassage d'idées de son époque, elle est à la recherche d'un renouveau continuel. Ainsi, la littérature étrangère occupe une place centrale dans sa stratégie éditoriale, en particulier la littérature francophone devient un pilier central pour le renouvellement et en plus elle rend cette maison d'édition spécialiste en francophonie. En effet, dans sa présentation officielle, Le Seuil se flatte de publier beaucoup d'auteurs francophones : Mouloud Feraoun, Mohammed Dib, Kateb Yacine, Aimé Césaire, Léopold Sédar Senghor, Anne Hébert, Jacques Godbout, puis Nelly Arcan, Ahmadou Kourouma, Alain Mabanckou ou Charif Majdalani. Toutefois, au delà de la conception déclarée, il faut remarquer que l'œuvre analysée, dans un premier temps, a été refusée par deux maisons d'éditions françaises, Le Seuil comprise, et a été publiée au Canada en 1968. Cet événement souligne que 
l'ouverture à la variation linguistique et culturelle liée à la francophonie et l'admission d'œuvres africaines francophone représentent un fait nouveau dans la culture française hexagonale, souvent soutenu par des agents externes. La traduction italienne n'apparait qu'en 1996, presque trente années après sa parution en France, dans la maison d'édition edizioni e/o. Cette maison existe depuis les années 70 . Sa politique éditoriale a toujours été très ouverte aux cultures étrangères : dès sa création, elle cherche à jeter des ponts, à ouvrir des «frontières littéraires» et à favoriser le dialogue entre les cultures. La preuve en est que cette maison d'édition a commencé par importer les littératures de l'Europe de l'Est au moment historiquement difficile de la Guerre Froide qui favorisait leur dénigrement ou leur instrumentalisation politique. La maison d'édition a ensuite concentré sa recherche sur la littérature américaine et les espaces considérés marginaux par la plupart des éditeurs italiens. Cette recherche de nouveaux domaines amène la maison d'édition à se vanter d'avoir découvert des auteurs de grand intérêt et faisant preuve d'originalité qui proviennent de l'hémisphère sud (par exemple le Cubain Pedro Juan Gutiérrez ou les Africains Chinua Achebe et Abasse Ndione) et qui sont alors inconnus du grand public italien.

Très éloignés de la culture-cible et représentatifs de la nouveauté, les romans de Kourouma sont classés par la maison d'édition dans une sous-série au nom qui évoque des pays lointains et exotiques : I Leoni- dal Mondo ("Les Lions- du Monde entier"); la sous-série comprend des œuvres méconnues en Italie qui sont originaires de la littérature africaine et antillaise contemporaines. La politique éditoriale de la maison d'édition et la place du roman dans une sous-série spécifique souligne que l'œuvre est perçue comme exotique et nouvelle, à l'écart des grands thèmes de la littérature et des grands courants culturels. En plus, aucune référence à la langue source ou à une communauté francophone n'est faite explicitement pour mieux souligner l'altérité de l'œuvre. Les traces d'un lien avec la francophonie et la langue française manquent totalement. Dans le classement dans la sous-série et dans les pages couvertures, aucune préface n'est donnée. Le roman est classé comme appartenant à une culture lointaine et exotique qui ne présente aucun lien avec l'Europe et la langue française. De plus, les images sur la couverture renforcent l'idée d'une culture étrangère et lointaine qui n'est pas du tout assimilable ou intégrable dans la culture occidentale : dans l'œuvre source, l'image représente un tissu aux couleurs très vives et une main noire tatouée comme référence au pagne et à des traditions locales supposées; dans le livre traduit en italien, l'image représente un contexte colonial qui pourrait se référer également à l'Afrique ou aux Antilles, il s'agit en particulier d'un vieil homme noir au chapeau de paille qui travaille dans une plantation de bananes. On passe d'un désir de faire connaittre la spécificité du pays africain à une vague image stéréotypée ayant une connotation exotique.

\section{2) La langue et les régionalismes employés dans Les Soleils des indépendances}

Le choix du français pour écrire un roman africain peut être justifié par plusieurs facteurs. En premier lieu, il faut considérer le problème du public : un roman en malinké n'aurait pas assez de lecteurs et il n'irait pas plus loin que sa diffusion locale, restant inconnu au réseau éditorial européen et nord-américain. Les idées véhiculées par le roman n'auraient jamais rejoint les grands centres de diffusion et de rayonnement culturel. En effet, les œuvres écrites dans une des nombreuses langues locales sont peu accessibles : le statut et le manque de normalisation de ces langues souvent orales, le nombre réduit des locuteurs, un système éducatif en français et la présence limitée des maisons d'éditions sur le territoire ne favorisent que l'écriture et la diffusion d'œuvres en français.

Un autre facteur qui motive le choix d'écrire le roman en français au lieu du malinké, réside dans la compétence linguistique de Kourouma qui affirme que sa connaissance du «malinké n'est pas assez développée » (Magnier 12); par conséquent, même s'il " pensait en malinké » (Magnier 12), son public virtuel l'oblige à écrire en français, bien qu'influencé par le malinké. Kourouma manifeste des réalités ou des façons de voir le monde propres au malinké dans une langue coloniale avec laquelle une distance 
culturelle évidente existe.

La langue de l'auteur est donc le français; toutefois, il ne s'agit pas de la forme standard parce que le français classique serait incapable, d'après l'auteur, de traduire la vision du monde d'un malinké; l'auteur lui-même affirme dans un entretien : "j'ai pensé en malinké et écrit en français en prenant une liberté que j'estime naturelle avec la langue classique » (Koné 83). Aussi le français employé dans ce roman estil plutôt une langue véhicule du métissage, une langue d'emprunt modelée afin que la culture malinké s'insère en produisant un double code de communication : dans la syntaxe, les structures des deux langues s'assimilent parfois et créent une structure linguistique nouvelle. À l'occasion, l'écrivain explique les mots ou la culture malinké à son récepteur de langue française par l'emploi de gloses intratextuelles et par des parenthèses. Du point de vue lexical, les nombreux emprunts, régionalismes et calques n'ont pas seulement la valeur de communiquer un signifié ou de désigner un référent spécifique, ils acquièrent dans leur connotation la valeur adjointe d'ethnicité et d'exotisme. La langue « bouleverse le lecteur de langue française grâce à ses structures grammaticales et syntaxiques irrégulières, et donne l'impression aux Africains francophones que l'écrivain a soumis cette langue à un procédé d'assimilation avec le malinké, sa langue maternelle » (Moro 355). Le français de Kourouma est souvent considéré comme un langage métis, parce que «la pensé malinké de Kourouma crée sa propre forme d'expression linguistique à l'intérieur de la langue française qui subit plusieurs changements structurels » (Moro 362). Dans le corpus considéré, parmi les changements structurels, on relève ainsi : l'emploi transitif des verbes que le français de référence considère comme intransitifs, l'emploi absolu des verbes transitifs ${ }^{1}$, le changement de diathèse verbale et l'hypostase, en particulier l'emploi des substantifs à la place d'adjectifs ou l'emploi adverbial des adjectifs. Le français de Kourouma peut être défini comme une interlangue littéraire qui reproduit le mélange linguistique complexe des pays africains francophones; il introduit dans son langage littéraire des néologismes et des emprunts qui font partie de l'usage local de la langue française. Du point de vue lexical, Les Soleils des Indépendances se caractérise par des régionalismes, des néologismes internes et de nombreux calques qui recouvrent tout type de diatopismes ${ }^{2}$ en synchronie (formel, sémantique, grammatical, de statut) (voir Annexes - Tableau 1). La catégorie des diatopismes sémantiques à l'intérieur des néologismes internes a souvent intéressé les critiques littéraires plus que les autres catégories. En effet, Gassama (25) fait référence à ce type de diatopisme quand il décrit comment l'auteur, pour créer son style, passe par trois étapes : dans la première, l'auteur désémantise le mot en le vidant de sa substance, ainsi, il dérange le lecteur dans l'univers linguistique qui lui est familier; dans une seconde étape, l'auteur charge le mot de nouvelles valeurs qui laissent une impression de flou en suscitant la curiosité du lecteur; enfin, l'auteur replace le lecteur dans son univers linguistique habituel et lui permet de prendre connaissance des nouvelles valeurs que le mot véhicule; la complicité auteurlecteur se fonde sur la «trahison» de la langue d'emprunt et sur le recours aux mécanismes d'expressivité de la langue maternelle du romancier.

Ce phénomène se produit dès le début, à partir du titre du roman (voir exemple 1), où Kourouma assimile la valeur sémantique du mot ère à celle de soleil en créant un lien entre les deux et en enrichissant le signe soleil d'une signification nouvelle qu'il puise dans sa langue malinké.

\section{Exemple 1 : « soleil»}

\footnotetext{
${ }^{1}$ Le non-respect des normes de transitivité-intransitivité du français d'Afrique est l'un des piliers de l'analyse de Manessy. ${ }^{2}$ Par diatopisme je désigne toute variante topolectale du français. Pour l'étude présente seulement le lexique a été pris en compte, il s'agit donc de prendre en considération les lexèmes qui changent de forme ou de signifié par rapport au français standard de référence et les néologismes par emprunt, calque ou innovation interne. Poirier (Poirier 43) propose une classification des diatopismes qui suit deux axes : un axe vertical, qui distingue les emplois selon leur origine, et un axe horizontal, qui spécifie la nature des phénomènes considérés. Suivant ce principe, les diatopismes du roman ont été classifié dans le tableau 1 en annexe.
} 
a) «Les Soleils des indépendances. » (Kourouma, Soleils- titre)

« I soli delle indipendenze. » (Kourouma, Soli- titolo)

b) «[...] si le défunt était de caste forgeron, si l'on n'était pas dans l'ère des Indépendances, (les soleils des Indépendances disent les malinkés), je vous le jure, on n'aurait jamais osé l'inhumer dans une terre lointaine et étrangère. » (Kourouma, Soleils 9)

«[...] se il defunto fosse stato della casta dei fabbri, e se non ci si fosse trovati nell'epoca delle Indipendenze (i soli delle Indipendenze, dicono i malinké) vi posso giurare che nessuno avrebbe mai osato seppellirlo in terra lontana e straniera. » (Kourouma, Soli 7-8)

c) «Balla aimait les raconter et d'un bout à l'autre d'un large soleil sans se répéter il assommait [...] les palabreurs. » (Kourouma, Soleils 123)

«A Balla piaceva raccontarle e, dall'inizio alla fine di un'intera giornata, senza ripetersi, stordiva gli ascoltatori. » (Kourouma, Soli 144)

Le mot soleil n'est pas employé ici dans son acception occidentale, cet emploi est un calque d'une langue africaine (Lafage s.v. solei). Soleil est calqué sémantiquement sur le mandékan téré (Blédé 38), et présente une signification polysémique, il prend des significations temporelles de dimension variable selon le contexte : au singulier, il signifie "astre », au pluriel, il correspond à la journée (surtout s'il est précédé par un adjectif numéral cardinal) ou à une période plus longue quand il est suivi du nom commun ou propre (exemple 1.a) jusqu'à côtoyer la notion d'espace. La première fois que le mot apparaît dans l'œuvre source, (exemple b) il est mis entre parenthèses et il est présenté par l'auteur comme une sorte d'équivalent avec le mot français ère; donc, l'auteur guide la compréhension des exemples suivants. Traduction : en italien le mot sole ne signifie «jour, journée » que dans le langage poétique; le mot sole est maintenu dans la traduction du titre, où la traduction du calque du mandekan n'est plus ressentie par le lecteur comme étranger mais comme poétique. Toutefois, à l'intérieur du texte le mot est parfois spécifié comme dans l'exemple 1.c quand il a le sens de «journée ». Le fait que les diatopismes les plus nombreux sont des néologismes internes (exemple 2), c'est-à-dire des innovations lexicales à partir des ressources propres de la langue en question, et des calques souligne la créativité linguistique soutenue qui est considérée comme une des caractéristiques principales du français en Afrique.

\section{Exemple 2 : démarabouter (se)}

« [...] sûrement là-haut le soleil avait réussi à se dépêtrer, à se démarabouter. » (Kourouma, Soleils 163)

«[...] sicuramente là in alto il sole era riuscito a svincolarsi e a fare capolino. » (Kourouma, Soli 152)

Il s'agit du verbe pronominal préfixé dé- + marabouter qui signifie "vaincre, s'en sortir" (sens figuré). Le verbe de base désigne des pratiques magico-religieuses faites par les marabouts ou le recours à ces pratiques pour vaincre un mauvais sort. Au sens figuré, il est glosé (Lafage s.v. marabouter) et il prend le signifié de "vaincre des obstacles puissants». L'auteur établit un lien avec les forces magiques par l'emploi de ce verbe. Traduction : périphrase assez fréquente en italien standard, fare capolino, qui signifie «pencher la tête partiellement d'un abri, de façon furtive et prudente ». Dans son usage figuré, on établit un lien avec le monde de la nature, des fleurs en particulier, parce que le mot italien capolino est un terme de la botanique qui désigne un type d'inflorescence.

Les néologismes par emprunt, c'est-à-dire les innovations lexicales par intégration d'un mot provenant 
d'une autre langue (exemple 3), sont moins nombreux mais néanmoins importants parce qu'ils représentent pour la plupart des diatopismes formels lexématiques, c'est-à-dire que la particularité diatopique concerne un lexème entier dans sa forme et dans son sens. Sous cette catégorie, les emprunts aux langues africaines sont les plus nombreux, même sans considérer la catégorie des toponymes, anthroponymes et ethnonymes. Une partie des emprunts provient des langues dites «de culture», c'est-à-dire des langues comme l'arabe ou les langues européennes qui ont un haut degré de normalisation, de standardisation et de statut et qui sont employées dans tous les domaines.

\section{Exemple 3 : balafon $*^{3}$}

«[...] contournons les danses : yagba, balafon, n'goumé. » (Kourouma, Soleils 143)

« [...] sbrighiamo in fretta le danze : yagba, balafon, n'goumbé.» (Kourouma, Soli 135)

Le balafon est une sorte de xylophone africain constitué de lames de bois de longueur différente. Des calebasses évidées forment la caisse de résonance. Variantes : balafon, balafong, balaphon. Il s'agit d'un composé d'origine mandenkan signifiant «xylophone + jouer» (Lafage s.v. balafon, IFA s.v. balafon). Le signifié du mot est présent dans le texte par l'emploi d'un hyperonyme qui le précède : danse. La traduction adopte la même technique de l'emprunt précédé d'un hyperonyme, le mot est donc maintenu dans sa forme et son signifié africains.

Plus de la moitié des régionalismes employés par l'auteur (38/75) est attestée dans l'IFA, c'est-à-dire qu'ils sont connus et fréquents dans toute l'Afrique francophone, en particulier en Afrique noire. Il s'agit donc de mots qui ne sont pas spécifiques seulement de la Côte-d'Ivoire. Les archaïsmes sont peu nombreux, contrairement à d'autres aires francophones qui ont connu une colonisation plus ancienne et plus profonde. Une réelle continuité du mot archaïque est difficile à démontrer dans ce secteur; l'hypothèse qu'il s'agit de néologismes aboutissant au même résultat d'un mot ancien est à prendre en considération. Le tableau récapitulatif inséré en annexes (tableau 1), inspiré par la grille proposée pour le français du Québec par C. Poirier (43) et adaptée par A. Thibault (Thibault section "Cours de francophonie $3^{e}$ année de licence, introduction ») exemplifie le classement de tous les diatopismes présents dans le roman étudié. La grille est un outil important pour une étude de la traduction orientée vers la langue source. Les exemples ont été traités en corpus parallèle; chaque entrée est représentée par le mot dans la langue source et par sa traduction.

\section{3) Techniques de traduction employées}

Dans la traduction italienne, plusieurs techniques sont utilisées. Le tableau 2 en annexe met en évidence les stratégies de traduction employées dans le texte étudié du point de vue de la langue cible. Les emprunts et les calques sont très nombreux, mais leur forme graphique ou typographique, dans la plupart des cas, change d'une langue à l'autre (voir exemples 4 et 5).

\section{Exemple 4 : Dioula}

« [...] la liberté du négoce qui fait le grand Dioula, le Malinké prospère. » (Kourouma, Soleils 11)

«[...] la libertà del traffico che rende grande il diula e prospero il malinké. » (Kourouma, Soli 21)

\footnotetext{
${ }^{3}$ Le signe diacritique * souligne que le type lexical est fréquent non seulement en Côte-d'Ivoire mais en français d'Afrique en général, en effet il est répertorié dans l'IFA et peut être relativement connu en français standard.
} 
Dioula désigne un commerçant africain musulman originaire du nord de la Côte-d'Ivoire et qui, en général, parle une langue du groupe mandé. Variantes : dioula, djoula, dyoula, dyula, djula, jula (IFA s.v. dioula; Lafage s.v. dioula). La référence à l'activité du Dioula est nette dans l'exemple. Dans la traduction, le mot est préservé mais avec adaptation au système de correspondances graphiques de l'italien : substitution du digramme $\langle o u\rangle$ par le graphème $\langle u\rangle$, changement typographique de la première lettre du majuscule au minuscule.

\section{Exemple 5 : tara}

«Fama et ses deux femmes occupaient la petite pièce avec un seul lit de bambou, un seul 'tara'.» (Kourouma, Soleils 151)

"Fama e le sue due mogli occupavano la stanzetta con un solo letto di bambù, un solo "tara". » (Kourouma, Soli 141)

Tara est un emprunt du mandekan qui signifie «lit traditionnel bas, fait de tiges ou de lattes entrecroisées et attachées par des fibres végétales ou du cuir » (Lafage s.v. tara; IFA s.v. tara). Le mot est précédé d'une glose dans le texte, « lit de bambou » et il est signalé comme étrange par l'auteur même grâce au procédé typographique des guillemets. La traduction italienne maintient le même procédé (glose et emploi des guillemets), seul le type de guillemets change. Il faut souligner que souvent, surtout dans le cas des noms propres et des toponymes, des adaptations graphiques ont été employées. Il s'agit surtout d'intégrer le mot en suivant les règles d'écriture et de prononciation du système italien. Dans la plupart des cas, le son $[\mathrm{u}]$ est rendu par le graphème $<\mathrm{u}>$ au lieu de $<\mathrm{ou}>$ de la langue source. Quand le lexème est mis en évidence par des procédés typographiques dans l'œuvre source, soulignant les limites du français standard de référence, le changement typographique est maintenu dans la traduction (exemple 5 «tara »/"tara"). Toutefois, l'œuvre cible contient une dizaine de mots faisant référence à la culture malinké qui subissent des changements typographiques : l'emploi de l'italique, suivi ou non d'une note, est la technique la plus employée (voir exemple 6).

\section{Exemple 6 : dolo*}

«Le chef de Toukoro dormait, ivre de dolo au milieu de ses sujets. » (Kourouma, Soleils 98)

« Ma il capo di Tukoro dormiva, ubriaco di dolo, tra i suoi sudditi. » (Kourouma, Soli 92)

Le dolo est une «boisson alcoolisée obtenue par fermentation d'une décoction de mil, de sorgho ou même de maïs ». Variantes : dolo, doro, $b$ d'lo, g'lo (IFA s.v. dolo; Lafage s.v. dolo). Le mot provient du mandekan ( $1^{\text {re }}$ attestation : 1892 (Duponchel s.v. dolo)). La traduction maintient l'emprunt qui est mis en évidence par le procédé typographique de l'italique. L'effet d'exotisme est donc ressenti plus fortement par un lecteur italien que par un lecteur francophone exogène. Les calques (voir exemple 7) sont moins nombreux et peuvent être motivés par la volonté esthétique de respecter les images de l'original. Le seul cas qui ne soit pas reconductible à cette motivation est donné par le calque en traduction de vers de Guinée (voir exemple 8).

\section{Exemple 7 : branches-épouvantails}

«Le vent léger soufflait le brûlis de la savane avec des sautes d'une puanteur insupportable et faisait craquer les feuilles des branches-épouvantails. » (Kourouma, Soleils 116)

«Il vento leggero soffiava sui terreni bruciati della savana con folate improvvise di un fetore 
insopportabile, facendo scricchiolare le foglie dei rami-spauracchio. » (Kourouma, Soli 109)

La locution composée par deux noms (branche + épouvantail) avec trait d'union est un néologisme de l'auteur qui établit une similitude entre les branches d'un arbre et un épouvantail. Le second segment connote la tête du composé, épouvantail « objet placé dans les champs [...] pour effrayer les oiseaux et les empêcher de manger les grains, les fruits » (Le Robert s.v. épouvantail). La traduction italienne est un nom composé qui a été recréé par un calque des signifiés des deux parties du mot composé, le traducteur emploie le mot italien ramo, «branche », et spauracchio qui désigne l'épouvantail ou une chose en général qui effraie dans la culture-cible.

\section{Exemple 8 : ver de Guinée}

«Des vers de Guinée poussaient dans les genoux et sous les aisselles. » (Kourouma, Soleils 169)

«Piccoli vermi di Guinea si formavano nelle sue ginocchia e sotto le ascelle. » (Kourouma, Soli 157)

Il s'agit d'un nom courant de la filaire de Médine qui parasite l'homme. Celle-ci se localise généralement dans les membres inférieurs. Elle transmet la dracunculose à laquelle on fait allusion dans la langue courante, par glissement de sens, par la locution verbale avoir le ver de Guinée (Lafage s.v. ver). La traduction italienne est calquée mot à mot sur l'original sans ajout d'explications.

Si l'adaptation graphique peut être vue comme un moyen d'acculturation, une domestication (Venuti The Translator 42) qui réduit le vide entre les cultures, l'augmentation du marquage typographique dans le roman cible augmente l'hybridité de la langue et ajoute une connotation d'exotisme et de «couleur locale ». En outre, le lecteur qui ne connait pas le signifié de ces mots est ainsi amené à conduire des recherches intra- et extratextuelles pour les comprendre.

Le traducteur adopte aussi la technique de la substitution d'un régionalisme par un mot qui a des liens sémantiques plus faibles ou inexistants avec le texte de départ. On note des simplifications par l'emploi d'un hyperonyme (voir exemple 9) ou d'un hyponyme qui n'est pas stylistiquement marqué, mais le cas le plus intéressant concerne le changement de référent (voir exemple 10).

\section{Exemple 9 : cabrin*}

«[...] les aboiements des cabots après les cabrins pour les faire entrer [...] (Kourouma, Soleils 118)

«[...] l'abbaiare dei cani dietro le capre per farle rientrare $[\ldots] »$ (Kourouma, Soli 111)

Le cabrin est une "espèce de chèvre naine à pattes courtes, au nom scientifique de capra hircus, commune en zone forestière »; le nom est fréquent au pluriel aussi et dans ce cas il désigne un ensemble de chèvres, boucs et chevreaux (IFA s.v. cabri, Lafage s.v. cabri). Le terme est traduit par l'hyperonyme capre qui dans la langue cible signifie simplement « chèvre, mammifère ruminant »,

\section{Exemple 10 : canari*}

« [...] se recroquevilla entre de vieux canaris et un cabot galeux. » (Kourouma, Soleils 106)

« [...] si rannicchiò tra vecchie casseruole, insieme a un cane rognoso. » (Kourouma, Soli 99)

Le terme désigne un « récipient en terre cuite ». Il s'agit d'un emprunt au galibi, langue amérindienne 
des Caraïbes. Ce terme est également présent dans les Antilles, d'où il a été exporté en Afrique. La première attestation remonte à 1664 (TLF s.v. canari). Il est très fréquent en Afrique pour désigner une grande variété de récipients utilisés principalement pour conserver et transporter les aliments, localement fabriqués avec de l'argile, plus rarement en bois (IFA s.v. canari, Lafage s.v. canari). Dans l'œuvre source, le mot est parfaitement intégré dans le texte. La traduction italienne consiste en une substitution par un mot fréquent en italien qui désigne un type commun de casserole utilisé en cuisine et qui joue le rôle d'équivalent culturel.

Si dans certains exemples le traducteur semble chercher un équivalent culturel (voir exemples 11 et 12 calebasse/grande zucca), dans d'autres, on peut difficilement expliquer le changement de référent (voir exemple 13).

\section{Exemple 11 : assise des repas}

«Et chaque jour le cercle autour des calebasses de tô s'était élargi de camarades de classe d'âge qui avaient choisi l'heure de l'assise des repas pour venir saluer.. » (Kourouma, Soleils 127)

«E ogni giorno il cerchio attorno alle grandi zucche di tô si era allargato ai coscritti che avevano scelto l'ora di pranzo per venire a salutare. » (Kourouma, Soli 119)

Assise est un mot d'origine ancienne (la première attestation remonte à 1170) qui désignait une assemblée, le plus souvent judiciaire. Par extension et métaphore, il est employé avec le signifié de "réunion de personnes ayant des préoccupations ou des goûts communs et se rencontrant régulièrement pour en débattre » (TLF s.v. assise). La tournure souligne la tradition d'une convivialité des repas que les membres de la famille consomment tous ensemble et en même temps en Afrique. La prise des repas donne lieu à un véritable cérémonial en Côte-d'Ivoire : les femmes déposent le repas contenu dans des calebasses, sur le sol, à côté du chef famille et disent « sùma sìgui ni béyi »; sùma « repas », sìgui «asseoir»: «le repas est assis » (Blédé 44). En utilisant cette expression, l'auteur souligne la nonexistence de la table dans la conception française : tous sont assis par terre, la main dans la même calebasse et mangent en communion. L'heure du repas est ainsi un moment d'unification (Blédé 44). Au lieu de maintenir le calque, la traduction italienne est raccourcie et simplifiée par l'emploi plus générique de ora di pranzo "l'heure du déjeuner »; l'image évoquée, le lien avec la tradition et l'idée d'exotisme ne sont pas véhiculés.

\section{Exemple 12 : calebasse*}

«[...] le repas s'asseyait autour des calebasses communes. » (Kourouma, Soleils 126)

« [...] il pranzo si serviva intorno alle grandi zucche comuni. » (Kourouma, Soli 118)

Ce lexème appartient au vocabulaire botanique. Il désigne en français standard le fruit du calebassier (Lagenaria siceraria, Crescentia cujete) dont l'écorce, séchée, sert de récipient et d'objet de décoration. En Côte-d'Ivoire, il désigne les récipients de forme et de taille très diverses obtenues des fruits de ces arbres (IFA s.v. calebasse; Lafage s.v. calebasse). La traduction consiste en une substitution par un équivalent culturel, zucche, qui, contrairement à calebasse, désigne un fruit commun en Europe (Cucurbita maxima) mais qui, lui aussi, peut être vidé et utilisé comme ornement; l'élément exotique est effacé par le non maintien de l'emprunt.

\section{Exemple 13 : cougal}


«Le cougal a été pris au piège, quelles raisons a le francolin de se jeter et rouler à terre en disant qu'il ne passera pas la nuit? » (Kourouma, Soleils 158)

« Ma, se il coguaro è stato preso in trappola, che ragioni ha il francolino di rotolarsi per terra dicendo che non passerà la notte? » (Kourouma, Soli 147)

Pour le mot congal qui désigne un « oiseau de la famille des Cuculiadae présent en Afrique » (Duponchel s.v. coucal; Lafage s.v. congal) dans la langue-source, le traducteur a opéré une substitution complète par un autre type d'animal, un mammifère plus connu dans la culture cible : le puma. En adoptant cette technique dans l'exemple 13, le traducteur maintient la métaphore de la victime, mais la rend moins explicite parce qu'un francolin n'est pas la victime privilégiée d'un puma. La substitution ou le choix d'un équivalent culturel différent, par exemple un autre oiseau rapace plus connu en Europe pour lequel un francolin serait la victime la plus typique, aurait pu donner un signifié plus évident et précis à la métaphore. Il est possible aussi que le traducteur n'ait pas trouvé le signifié du mot congal dans les dictionnaires de référence pour le français standard (Grand Robert, Trésor de la langue française) et qu'il l'ait interprété comme une faute de frappe du mot français cougar. Un cas pareil est représenté par la traduction du mot malinké toto (voir exemple 14) qui désigne le rat le plus connu localement qui vit sous terre et commet de nombreux vols dans les jardins potagers.

\section{Exemple 14 : toto}

«Elle ment comme une aveugle, comme une édentée, elle vole comme une toto.» (Kourouma, Soleils 129)

«Mente come una cieca, come una sdentata, ruba come un pidocchio. » (Kourouma, Soli 122)

Ce terme est en fait un emprunt du mandenkan qui désigne le Cricetomis gambianus, rat qui peut atteindre une taille de $50 \mathrm{~cm}$ avec une queue d'environ $50 \mathrm{~cm}$, au pelage brun-gris rude et assez clairsemé. Ce rat vole dans les jardins potagers aussi est-il est appelé rat voleur ou rat de Gambie (Lafage s.v. toto; IFA s.v. rat voleur). Dans les dictionnaires du français standard, la forme est présente mais avec le seul signifié de "pou" dans le langage populaire. C'est probablement pour cette raison que le traducteur emploie le mot pidocchio qui, en italien, ne désigne que le parasite. Par cette technique, au lieu d'utiliser un emprunt ou un hypéronyme, la similitude de l'exemple tiré de l'œuvre-source perd tout son sens en italien.

\section{Exemple 15 : plaisanterie (frère de)}

«Puis il y eut les griots (sauf Diamourou), les frères de plaisanterie qui réclamaient, et tous les autres qui gémissaient et tendaient les mains [...].»(Kourouma, Soleils 112)

«Poi arrivarono i griot (a parte Diamuru), i compagni di scherzi, e tutti gli altri che si lamentavano e tendevano la mano [...].» (Kourouma, Soli 119)

Cette notion renvoie à un type de lien ethnique, qui désigne une "personne liée à une autre (de famille ou d'ethnie différente) par la parenté à plaisanterie, ce qui lui interdit toute querelle avec cette personne et l'autorise au contraire à railler sans aucun ménagement »(Lafage s.v. frère- frère de plaisanterie). La traduction est une reprise partielle de l'expression formelle sans aucune note ou explication. Par cette technique le lecteur italien comprend que le référent dont l'auteur parle n'est qu'un ami, un copain avec qui rigoler. 
La traduction reprend partiellement l'expression formelle en changeant le mot frère par compagni, qui, sans aucune note ou explication, enlève tout lien de parenté et laisse inférer qu'il s'agit d'un simple groupe d'amis effaçant ainsi l'arrière-plan traditionnel. Ces changements de référent peuvent être interprétés comme des erreurs du traducteur ou comme l'évidence de la limite des outils employés comme les dictionnaires de français standard de référence.

L'amplification est très exploitée. Ce procédé se traduit par l'emploi de périphrases et de notes en bas de page (voir exemple 16). Le traducteur n'ajoute pas de gloses intratextuelles entre parenthèses ou virgules, il se limite à traduire celles déjà présentes insérées par l'auteur lui-même.

\section{Exemple 16 : viandé}

«Un carnage, une ripaille aussi viandée bouleversa toute la province. » (Kourouma, Soleils 139)

«Una simile carneficina, una tale abbuffata di carne sconvolse tutta la provincia. » (Kourouma, Soli 131)

Il s'agit d'un néologisme qui est en fait un adjectif dénominal dérivé de viande au signifié transparent de «plein de viande» (Lafage s.v. viandé). La traduction consiste en une substitution par la périphrase abbuffata di carne signifiant mot à mot « gueuleton de viande, repas abondant de viande ».

Deux types de notes par structure et fonction sont à relever dans le texte cible : le premier, le plus employé et le plus traditionnel, ajoute une explication au référent nommé par l'auteur (exemple 17), ainsi le texte est plus accessible au lecteur italien et l'étrangeté de la culture africaine dans laquelle l'événement se déroule est mise en évidence; le second type est une note extratextuelle qui a la fonction d'illustrer la technique de traduction adoptée par le traducteur (exemple18).

\section{Exemple 17 : boubou*}

«Les boubous blancs, bleus, verts, jaunes, disons de toutes les couleurs, moutonnaient, les bras s'agitaient et le palabre battait. » (Kourouma, Soleils 13)

«I lunghi boubou bianchi, verdi, gialli, praticamente di tutti i colori si accavallavano, mentre le braccia si agitavano e i discorsi fervevano.

(nota) lunga veste, caratteristica di molte popolazioni africane. » (Kourouma, Soli 11)

La première attestation date de 1867. Il s'agit d'un type lexical du malinké qui désigne un vêtement traditionnel ou moderne féminin. Porté surtout par les habitants du nord de la Côte-d'Ivoire, il peut être semblable au boubou de cérémonie : il est long, à manches amples avec une poche sur le devant (Lafage s.v. boubou; IFA s.v. boubou). Dans l'original, le mot est parfaitement intégré au texte, comme un mot du français standard. En revanche, la traduction maintient l'emprunt du malinké mais ajoute une note en bas de page qui explique le référent et précise qu'il appartient à la culture africaine en général.

\section{Exemple 18 :Toubab*}

« Avant la conquête des Toubabs [...].» (Kourouma, Soleils 18)

«La conquista da parte dei Bianchi [...].

(nota) Tubab: i Bianchi, questo termine, frequente nel testo, sarà reso con "Bianchi”"» (Kourouma, Soli 12) 
Il s'agit d'un emprunt probablement au wolof qui désigne toute personne à peau blanche non sémite, plus particulièrement le Français européen, surtout dans les régions du nord de la Côte-d'Ivoire. Il y a des variantes : tubabu, tibabu, toubabou, toubabesse. Il peut être employé avec une connotation péjorative (IFA s.v. toubab, Lafage s.v. toubab). La traduction ajoute une note qui se limite à déclarer la stratégie adoptée en traduction pour ce mot : Toubab sera remplacé par le mot bianchi qui en italien signifie «personne à peau blanche du groupe humain d'origine européenne ».

L'ajout de notes explicatives représente une altération de l'expérience de lecture pour le destinataire de la culture cible. En effet, le lecteur n'est pas forcé de chercher des explications en dehors du texte et fait une expérience de lecture plus fluide, le texte devient plus lisible et orienté vers la culture cible. Un lecteur francophone exogène, qui ne connait pas le milieu ivoirien, a les mêmes difficultés en lisant l'œuvre originale : augmenter le nombre de notes de bas de page par rapport au roman source représente l'avantage d'accroître la compréhension immédiate mais s'associe au désavantage de ne pas respecter l'effet plus ou moins exotique voulu par l'auteur. En outre, le lecteur de l'œuvre traduite devient ainsi moins actif dans son expérience de lecture en ce qui concerne la recherche des signifiés liés à la culture malinké.

Le traducteur ne produit pas une traduction totalement orientée sur la cible ou totalement orientée sur la source; il recourt à une variété de stratégies différentes qui sont parfois le signe d'une exotisation voulue (emprunts, changements typographiques), parfois d'une clarification recherchée (notes en bas de page). Grâce aux procédés de traduction employés, on peut dégager une tendance modérée vers le maintien de l'effet d'étrangeté à produire sur le lecteur et l'exotisation du texte. En effet, la langue africaine laisse des traces bien évidentes non seulement dans le français du roman source, mais dans le texte cible aussi; toutefois, elles sont plus isolées et mises en évidence par des procédés typographiques, des notes intégrées dans la langue italienne.

\section{Conclusion}

Les diatopismes dans le français de la Côte-d'Ivoire représentent la spécificité de la langue du roman et reflètent la situation sociolinguistique complexe de l'œuvre littéraire. L'emploi d'une langue parfois définie comme hybride par la critique souligne la volonté de l'auteur de rompre avec la langue des colons français et d'affirmer avec orgueil l'identité différente du colonisé. Aussi les diatopismes ne sont-ils pas seulement un simple trait de style qui donne une couleur locale, mais ils sont porteurs de signifiés et de valeurs profondes. La rébellion contre le colonisateur passe par l'appropriation et le changement radical de sa langue, ce qui est rendu possible grâce à l'apport de la langue du colonisé qu'on a cherché à anéantir. Une étude lexicale approfondie de la langue employée se révèle donc fondamentale; elle a été réalisée ici suivant la méthode et selon les outils de la lexicographie différentielle. Une telle analyse représente une contribution importante pour la mise en valeur de la richesse de la langue source, les valeurs qui lui sont associées et, donc, les spécificités à véhiculer dans la traduction. Dans la culture cible, la maison d'édition prend une position nette en faveur de l'exotisme de l'œuvre : le fait que le roman a été présenté dans une sous-série de la maison d'édition en quête de nouveaux domaines et de nouvelles cultures à transmettre en est une preuve. Toutefois, le traducteur n'opère pas des choix si nets, il utilise des techniques différentes qui oscillent entre une traduction orientée vers la source et une traduction orientée vers la cible. D'un côté, le traducteur maintient l'exotisme de l'œuvre par l'introduction d'emprunts et de calques; de l'autre côté, il banalise en cherchant des équivalents, des hyperonymes, des substitutions. L'introduction de notes compense l'emploi d'une langue plus standard par rapport à la source. Toutefois, cette technique rend la spécificité du roman plus explicite; elle contribue même à le rendre plus accessible, mais efface le sentiment d'étrangeté ressenti par le lecteur francophone exogène. La volonté de souligner l'exotisme du roman est évidente, toutefois le traducteur n'ose pas prendre une position trop nette au niveau de la langue en 
Alternative Francophone vol.1, 3(2010), 49-66

http://ejournals.library.ualberta.ca/index.php/af

faveur d'un registre et d'un lexique lointains de l'italien standard qui auraient provoqué un plus grand effet d'exotisme chez le lecteur italien. 


\section{Annexes}

Tableau 1 : classification des diatopismes employés dans le roman

Il s'agit souvent de mots à usage répété dans le roman Les Soleils des indépendances. Ils sont mis en parallèle avec la traduction italienne et classés suivant le tableau de Poirier (Poirier 43) réélaboré par Thibault (Thibault section « Cours de francophonie $3^{\text {ème }}$ année de licence, introduction »).

\begin{tabular}{|c|c|c|c|}
\hline & Archaïsmes & Néologismes par emprunts & $\begin{array}{l}\text { Néologismes internes et } \\
\text { calques }\end{array}$ \\
\hline \multicolumn{4}{|l|}{$\begin{array}{l}\text { Diatopismes } \\
\text { formels : } \\
\text { phonétiques et } \\
\text { phonologiques }\end{array}$} \\
\hline \multirow[t]{4}{*}{$\begin{array}{l}\text { Diatopismes } \\
\text { formels : } \\
\text { lexématiques }\end{array}$} & \multirow[t]{4}{*}{$\begin{array}{l}\text { méfaire/comp } \\
\text { ortarsi } \\
\text { scorrettamente }\end{array}$} & $\begin{array}{l}\text { Arabe et langues de culture : } \\
\text { «alphatia»/ "alphatia"; } \\
\text { "bissimilai" / "bissimilai"; } \\
\text { cabrin/capre; chéchia/chéchia + } \\
\text { note; Talibet/talibet; }\end{array}$ & \multirow{4}{*}{$\begin{array}{l}\text { bubulement/verso; cabrin/capre; } \\
\text { déhonté/sconcio, svergognato; } \\
\text { démarabouter/fare capolino; } \\
\text { fourmi magna/ formica gigante; } \\
\text { grillotement/sfrigolio; } \\
\text { maraboutage/tresca religiosa; } \\
\text { tutubement/verso; } \\
\text { viandé/abbuffata di carne; }\end{array}$} \\
\hline & & $\begin{array}{l}\text { Langue africaines : } \\
\text { balafon/balafon; } \\
\text { boubou/boubou + nota; } \\
\text { cauris/cauri; cougal/coguaro; dja } \\
\text { (le double)/dja (il doppio); } \\
\text { dolo/dolo; fonio/fonio; fourmi } \\
\text { magna/formicha gigante; } \\
\text { foutou/futu; } \\
\text { gnamokodé/gnamokodé; } \\
\text { Houmba/Humba; périphrase + } \\
\text { kala/périphrase + kala; } \\
\text { kapok/kapok; Koma/Koma; } \\
\text { n'goumbé/n'goumbé; ni (l'âme)/ } \\
\text { ni (l'anima); «tara»/"tara"; tô/tô; } \\
\text { toto/pidocchio; toubabs/ i } \\
\text { Bianchi + nota; yagba/yagba; }\end{array}$ & \\
\hline & & $\begin{array}{l}\text { Toponymes, anthroponymes, } \\
\text { ethnonymes : Dioula/ dioula; } \\
\text { Djoliba/djoliba; Fama } \\
\text { Doumbuya/Fama Dumbuya; } \\
\text { Foula/Foula; } \\
\text { Horodougou/Horodugu; } \\
\text { malinké/malinké + nota; } \\
\text { Ouassoulou/Uassulu; } \\
\text { Oulofs/ulof; } \\
\text { Togobala/Togobala; }\end{array}$ & \\
\hline & & $\begin{array}{l}\text { Origine inconnue ou d'autre } \\
\text { origine : canari/canari; griot/ }\end{array}$ & \\
\hline
\end{tabular}




\begin{tabular}{|c|c|c|c|}
\hline & & $\begin{array}{l}\text { griot + nota; lougan/ } \varnothing ; \\
\text { margouillat/insetto; }\end{array}$ & \\
\hline $\begin{array}{l}\text { Diatopismes } \\
\text { formels : } \\
\text { phraséologiqu } \\
\text { es }\end{array}$ & & Heure de l'ourebi/ ora dell'urebi; & $\begin{array}{l}\text { assise des repas/ora di pranzo; } \\
\text { asseoir le deuil/presiedere il lutto, } \\
\text { trascorrere il lutto; asseoir la } \\
\text { palabre/fissare un incontro; } \\
\text { branche-épouvantail/rami- } \\
\text { spauracchio; courber (une } \\
\text { prière)/osservare (una preghiera); } \\
\text { creuseur de trous de } \\
\text { rats/scavatore di tane di topi; } \\
\text { durcir les oreilles/rifiutare di } \\
\text { ascoltare; frères de } \\
\text { plaisanterie/compagni di scherzi; } \\
\text { salive incantatoire/saliva magica; } \\
\text { vers de Guinée/vermi di Guinea; }\end{array}$ \\
\hline $\begin{array}{l}\text { Diatopismes } \\
\text { sémantiques }\end{array}$ & & & $\begin{array}{l}\text { battre/cadere; cassé/incapace; } \\
\text { dévirilisé/impotente; } \\
\text { éclater/diramare, ricominciare a } \\
\text { splendere, saltare agli occhi; } \\
\text { excision/escissione+ nota; } \\
\text { marabout/marabutto; } \\
\text { palabreur/ascoltatori; salueur/la } \\
\text { gente per salutarlo; soleils/soli, } \\
\text { giornata; vidage/pulizia; }\end{array}$ \\
\hline $\begin{array}{l}\text { Diatopismes } \\
\text { grammaticaux }\end{array}$ & & & $\begin{array}{l}\text { Tournures syntaxiques : } \\
\text { transitivisation des verbes } \\
\text { intransitifs; l'emploi absolu des } \\
\text { verbes; hypostase ou changement } \\
\text { de classe grammaticale; }\end{array}$ \\
\hline $\begin{array}{l}\text { Diatopismes } \\
\text { de statut }\end{array}$ & $\begin{array}{l}\text { palabreur/conv } \\
\text { ersatore, } \\
\text { ascoltatore; }\end{array}$ & $\begin{array}{l}\text { Harmattan/ harmattan + nota; } \\
\text { marigot/palude; pagne/pagne; }\end{array}$ & $\begin{array}{l}\text { calebasse/grande zucca; } \\
\text { charognards/avvoltoi; } \\
\text { flamboyant/flamboyant + nota; } \\
\text { fromager/eriodendro; } \\
\text { hivernage/hivernage/stagione } \\
\text { delle piogge + nota; } \\
\text { jujubier/giaggiolo; } \\
\text { maraboutage/tresca religiosa; } \\
\text { marabout/marabutto; } \\
\text { marabouter/darsi da fare con le } \\
\text { autorità islamiche; }\end{array}$ \\
\hline
\end{tabular}




\section{Tableau 2 : techniques de traduction employées}

Le schéma suivant (réadapté de Taylor 2000) met en évidence les stratégies de traduction employées dans le texte étudié du point de vue de la langue cible.

\section{Equation :}

\section{- Emprunts}

\begin{tabular}{|c|c|}
\hline $\begin{array}{l}\text { Adaptation graphique qui } \\
\text { intègre l'emprunt dans la } \\
\text { langue cible }\end{array}$ & $\begin{array}{l}\text { Fama Doumbuya/Fama Dumbuya; Dioula/diula; foutou/futu; } \\
\text { Horodougou/Horodugu; Houmba/Humba; Ouassoulou/Uassulu; } \\
\text { Oulofs/ulof; ourebi/urebi; }\end{array}$ \\
\hline \multirow{3}{*}{$\begin{array}{l}\text { Changement } \\
\text { typographique } \\
\text { (emploi différent des } \\
\text { guillemets, de l'italique et } \\
\text { de la majuscule) }\end{array}$} & |«alphatia»/“alphatia”; «tara»/"tara"; \\
\hline & $\begin{array}{l}\text { chéchia/chéchia + note; dolo/dolo; kala/kala; ni (l'âme)/ni (l'anima); dja (le } \\
\text { double)/dja (il doppio); heure de l'ourebi/ ora dell'urebi; }\end{array}$ \\
\hline & Dioula/diula; Djoliba/djoliba; Talibet/talibet; \\
\hline $\begin{array}{l}\text { Maintien graphique et } \\
\text { typographique intégral de } \\
\text { la forme }\end{array}$ & $\begin{array}{l}\text { "bissimilai"/”bissimilai”; fonio/fonio; Foula/Foula; } \\
\text { gnamokodé/gnamokodé; kapok/kapok; Koma/Koma; pagne/pagne; } \\
\text { tô/tô; Togobala/Togobala; balafon/balafon; n'goumbé/n'goumbé; } \\
\text { yagba/yagba; }\end{array}$ \\
\hline Emprunt sémantique & soleil/soli; \\
\hline $\begin{array}{l}\text { Adaptation } \\
\text { morphologique }\end{array}$ & Oulofs/ulof; \\
\hline
\end{tabular}

- Calques de traduction

branches-épouvantails/rami spauracchio; creuseur de trous de rats/scavatore di tane di topi; salive incantatoire/saliva magica; vers de Guinée/vermi di Guinea;

- Équivalents

épaté/sciancato; fromager/eriodendro; asseoir le deuil/presiedere il lutto, trascorrere il lutto; asseoir la palabre/fissare un incontro; durcir les oreilles/rifiutare di ascoltare;

\begin{tabular}{l|l} 
Étymologiquement apparentés & cauris/cauri; kaolin/caolino; marabout/marabutto;
\end{tabular}

Substitution : le mot employé dans le texte cible a une relation sémantique ou morpho-syntaxique avec le texte source plus faible ou inexistante.

- Changement de référent par équivalent culturel ou possible incompréhension du traducteur

canari/casseruola; cougal/coguaro; toto/pidocchio; l'assise des repas/ora di pranzo; grillotement/sfrigolio; jujubier/giaggiolo; calebasse/grande zucca; frères de plaisanterie/compagni di scherzi; 
- Hyperonyme

cabrin/capra; marigot/palude; charognards/avvoltoi; bubulement/verso; tutubement/verso; clabaud/cane;

- Co-hyponymes non marqués stylistiquement

Fourmi magna/ formica gigante; déhonté/sconcio, svergognato; palabreur/conversatore, ascoltatore; dévirilisé/impotente; soleils/soli; courber (une prière)/osservare (una preghiera); battre/cadere; cassé/incapace;[avoir] finir/finire, morire; éclater/diramare; vidage/pulizia;

Amplification : le traducteur ajoute du matériel au texte source afin d'augmenter la compréhensibilité du texte cible.

- Ajout d'une note de bas de page

\begin{tabular}{|l|l|}
\hline $\begin{array}{l}\text { Notes explicatives du } \\
\text { référent }\end{array}$ & $\begin{array}{l}\text { boubous/boubou + lunga veste, caratteristica di molte popolazioni } \\
\text { africane; chéchia/chéchia + tradizionale copricapo in lana di origine } \\
\text { maghrebina; griot/griot + artisti della parola : cantastorie, buffoni o } \\
\text { narratori, a loro è spesso affidata la trasmissione del patrimonio } \\
\text { tradizionale orale. Possono essere, a seconda dei gruppi di appartenenza, } \\
\text { rispettati e temuti o discriminati e scherniti; malinké/malinké + popolo } \\
\text { appartenente alle genti madingo, la cui cultura è legata a stati e imperi che } \\
\text { hanno fatto la storia dell'Africa. Sono per lo più stanziali sulle due rive del } \\
\text { medio Niger, ma la loro eccezionale abilità negli scambi li ha portati nel } \\
\text { tempo a migrazioni e spostamenti; excision/escissione+ escissione o } \\
\text { clitoridectomia; intervento "chirurgico" praticato come fondamentale } \\
\text { momento iniziatico (circoncisione femminile) da alcuni popoli musulmani; } \\
\text { flamboyant/flamboyant + alberi dai fiori rosso vivo; }\end{array}$ \\
\hline $\begin{array}{l}\text { Notes extratextuelles, } \\
\text { explicatives de la technique } \\
\text { de traduction employée }\end{array}$ & $\begin{array}{l}\text { harmattan/harmattan + vento caldo e secco; il termine indica anche la } \\
\text { stagione secca, contrapposto a hivernage, tradotto con "stagione delle } \\
\text { piogge; Toubabs/ i Bianchi + Tubab: i Bianchi, questo termine, frequente } \\
\text { nel testo, sarà reso con "Bianchi"; }\end{array}$ \\
\hline
\end{tabular}

- Périphrase

viandé/abbuffata di carne; méfaire/comportarsi scorrettamente; maraboutage/tresca religiosa; se démarabouter/fare capolino; hivernage/stagione delle piogge; nuiter/trascorrere la notte; éclater/ricominciare a splendere, saltare agli occhi; se marabouter/darsi da fare con le autorità islamiche; salueur/la gente per salutarlo;

- Ajout

marmaille/già con una prole più numerosa di due nidiate di topi; 
Réduction : le mot a été omis.

- élimination

lougan/Ø;

Réorganisation : la syntaxe est réorganisée selon la langue cible

Tournures syntaxiques, proverbes, maximes.

Exemple : Pourquoi tourner le dos à tout cela pour marcher un mauvais voyage?/Perché voltare le spalle a tutto ciò per intraprendere un cattivo viaggio?; il n'avait pas soutenu un petit rhume/non era riuscito a sopportare un raffreddorino 


\section{Bibliographie}

ATILF : Trésor de la langue française informatisé (TLF). Web. 1 Juin 2010.

Blédé, Logbo. Interférences linguistiques dans "Les soleils des indépendances" d'Ahmadou Kourouma. Paris : Publibook, 2006.

Duponchel, Laurent. Dictionnaire du français de la Côte-d'Ivoire. Abidjan : Université d'Abidjan, 1975.

Gassama, Makhily. La langue d'Ahmadou Kourouma, on Le francais sous le soleil d'Afrique. Paris : ACCT :Karthala, 1995.

IFA : équipe IFA, Racelle-Latin, Danièle (Ed.). Inventaire des particularités lexicales du français en Afrique noire. Paris : Edicef, 1988.

Koné, Amadou. «Le romancier africain devant la langue d'écriture : problèmes des relations entre la langue et l'identité. » Francofonia 22 (1992) : 75-88.

Kouassi Kouamé, Germain. Le phénomène de l'appropriation linguistique et esthétique en littérature africaine de langue française. Le cas des écrivains ivoiriens : Dadie, Kourouma, Adiaffi. Paris : Publibook, 2007.

Kourouma, Ahmadou. Les soleils des indépendances. Paris : Editions du Seuil, 1995.

Kourouma, Ahmadou (trad. italiana di Amari M.). I soli delle indipendenze. Roma : Edizioni e/o, 2004.

Lafage, Suzanne. «Le lexique français de Côte-d'Ivoire : appropriation et créativité. » Le français en Afrique 16-17 (2002). Nice : CNRS/Institut de linguistique française.

Magnier, Bernard (propos recueillis par). "Ahmadou Kourouma.»Culture Sud Notre librairie 87Littérature de Côte-d'Ivoire 2 (1987). Paris : ADPF-Culturesfrance.

Manessy, Gabriel. Le français en Afrique noire. Mythe, stratégies, pratiques. Paris : L'Harmattan. 1994.

Moro, Adriana. «La langue de la communication interculturelle. L'exemple de Les Soleils des indépendances et de Monné, outrages et défis d'Ahmadou Kourouma. »Les littératures africaines: transposition? Actes du colloque APELA septembre 2001. Ed. Gilles Teulié. Montpellier : Université Paul-Valéry Montpellier III, 2002, 355-368.

Poirier, Claude. «Les variantes topolectales du lexique français : Propositions de classement à partir d'exemples québécois. » Le régionalisme lexical. Ed. Francard et Danièle Latin-Racelle. Louvain-laNeuve : Duculot, 1995, 13-56.

Risterucci-Roudnicky, Danielle. Introduction à l'analyse des oeuvres traduites. Paris : A. Colin, 2008.

Taylor, Christopher. Language to language: a Practical and Theoretical Guide for Italian-English Translator. Cambridge : University Press, 2000.

Thibault, André. «Cours de francophonie 3e année de licence, introduction (2a, 2b). » Page d'accueil d'André Thibault. N.p., n.d.Web. 1er Août 2010.

Venuti, Lawrence. The Translator's Invisibility: a History of Translation. New York : Routledge, 1995.

Venuti, Lawrence. Retbinking Translation: Discourse, Subjectivity, Ideology. New York : Routledge, 1992. 